\title{
32-adrenoceptor blocker ICI118551 attenuates Pseudomonas aeruginosa corneal infection in mice
}

\author{
Xiubin $\mathrm{Ma}^{1}$, Fangying Song ${ }^{1}$, Qun Wang ${ }^{1}$, Ya $\mathrm{Li}^{1}$, Jing $\mathrm{Li}^{1}$, Shengqian Dou ${ }^{1}$, Lixin Xie ${ }^{1}$, \\ and qingjun $\mathrm{Zhou}^{1}$ \\ ${ }^{1}$ Qingdao Eye Hospital of Shandong First Medical University
}

October 5, 2020

\begin{abstract}
Purpose: Our previous studies have confirmed that the catecholamine norepinephrine (NE) promoted the corneal infection and progression of Pseudomonas aeruginosa (P. aeruginosa) keratitis. Here we explored the effects of adrenoceptor (AR) blockers on the severity of P. aeruginosa keratitis in mice. Methods: A total of $630 \mathrm{C} 57 \mathrm{BL} / 6$ mice were used and ocularly inoculated with P. aeruginosa (ATCC 19660). Six AR blockers were topically administrated $6 \mathrm{~h}$ before or combined with Tobrex $12 \mathrm{~h}$ after bacterial inoculation. Clinical scores, neutrophil infiltration and neutrophil extracellular traps (NETs), proinflammatory factors and bacterial virulence factors expression, bacterial load and biofilm formation were evaluated in vivo. The growth inhibitory and bactericidal activity of ICI118551 was measured in vitro. Results: Among various blockers, the selective $\beta 2$-AR blocker ICI118551 showed the most significant improvement in disease severity. Prophylactic administration of ICI118551 attenuated the clinical scores, neutrophil infiltration, NETs formation, proinflammatory factors expression of infected corneas, accompanied with the reduction of bacterial load, virulence factors expression, and biofilm formation. When adjunctive treatment with Tobrex, ICI118551 exhibited apparent therapeutic effects with the reduced severity after $12 \mathrm{~h}$ of bacterial inoculation. Moreover, ICI118551 inhibited bacterial growth and possessed bactericidal activity in vitro. In addition, ICI118551 had no significant influence on ocular barrier function and intraocular pressure. Conclusions: The selective $\beta 2-\mathrm{AR}$ blocker ICI118551 attenuated the severity of P. aeruginosa keratitis in mice, which may represent the potential prophylactic and therapeutic approach to control the P. aeruginosa corneal infection.
\end{abstract}

\section{Introduction}

Microbial keratitis is a kind of vision-threatening corneal disease usually accompanied by ocular injury or contact lens wear (Carntet al ., 2017; Fleiszig et al ., 2020; Lakhundi et al ., 2017). Once infected, it progresses rapidly despite appropriate antimicrobial therapy (Lakhundi et al ., 2017).Pseudomonas aeruginosa (P. aeruginosa ) is identified as a leading pathogen causing microbial keratitis worldwide (Carnt et al ., 2017; Lakhundi et al ., 2017; Suzuki et al ., 2018). Corneal ulcers due to P. aeruginosa are reported more destructive than other bacterial corneal ulcers, with significant potential for permanent vision loss (Oka et al ., 2015). These symptoms are attributed to the host's inflammatory response, bacterial toxins and exoproducts (Hazlett, 2004).

The pathogenesis of $P$. aeruginosa keratitis involves bacterial virulence factors and host excessive inflammatory response (Hazlett, 2004; Willcox, 2007). P. aeruginosa employs a serise of cell-associated and extracellular virulence factors such as pili, flagella, elastase, extoxin A, pyocyanin and biofilm, which can invade or kill corneal cells and induce corneal destruction (Willcox, 2007). Along with these virulence factors, the type-3 secretion system (T3SS) is involved in the pathogenesis of keratitis. The T3SS infuses toxins into host cells, immune evasion, and the Psl exopolysaccharide, which forms tenacious biofilms (Ma et al ., 2012; Thanabalasuriaret al ., 2019). The formation of biofilm plays an important role in antibiotic resistance and disease progression, which protects $P$. aeruginosa from the phagocytosis of neutrophils, and 
induces neutrophils extracellular traps (NETs) formation by neutrophils (Zhaoet al ., 2019). Although the infiltration of bacteria is prevented to some extent, the biofilm and NETs hinder the rapid killing of bacteria by neutrophils and prolongs the course of disease. The elastase produced by $P$. aeruginosa and neutrophils could cause corneal erosion (Thanabalasuriar et al ., 2019).

In addition to bacterial virulence factors, excessive activation of the host defense system is another important factor causing tissue destruction (Willcox, 2007; Yue et al ., 2016). The host inflammatory response can eliminate invading pathogens in the cornea (Lee et al ., 2015; Okada et al ., 2006; Papayannopoulos, 2018; Yang et al ., 2016), but also lead to tissue destruction (Willcox, 2007). A reduction of tissue destruction is accompanied by a significant decrease in recruitment of polymorphonuclear neutrophils (PMN) during bacterial infection in corneas of mouse (Pearlman et al ., 2008). The conventional drug therapies currently available for $P$. aeruginosa keratitis were antibiotics which would contribute to bacteria elimination (Austin et al ., 2017), but the resistance to antibiotics of $P$. aeruginosa is becoming increasingly via intrinsic and acquired mechanisms (Cunrath et al ., 2019; Subediet al ., 2018; Vazirani et al ., 2015), Therefore, the drug capable of controlling inflammatory response maybe can alleviate inflammation during $P$. aeruginosa corneal infection.

Our previous studies reported that both epithelial injury and extended contact lens wear induced corneal NE secretion. Elevated NE promoted the adhesion and biofilm formation of P. aeruginosa, and the infiltration of neutrophils into cornea, which further promoted the $P$. aeruginosa corneal infection and progression in mice (Liet al ., 2020; Ma et al ., 2020). In this study, we further investigated the effects of various adrenoceptor (AR) blockers on $P$. aeruginosa infection in mouse cornea, and found that the selective $\beta 2$-AR blocker ICI118551 attenuated the severity of $P$. aeruginosa keratitis in mice, which may represent the potential prophylactic and therapeutic treatment for $P$. aeruginosainfection in cornea.

\section{Methods}

\section{Animals, reagents, and antibodies}

The adult C57BL/6 mice (age, 8 weeks; weight, 20-25 g; amount, 630) were purchased from Beijing Vital River Laboratory Animal Technology Co., Ltd, which were maintained in the animal facility of Shandong Eye Institute. All procedures were carried out in accordance with the Association for Research in Vision and Ophthalmology (ARVO) Statement for the Use of Animals in Ophthalmic and Vision Research. The reagents and antibodies are listed in Supplementary Table 1.

\section{Infection procedure, clinical examination and application of}

\section{reagents}

Mice were anesthetized and the left corneas were scratched to create three 1-mm incisions using a sterile 25-gauge needle, then inoculated with bacterial suspension containing $1 \times 10^{5}$ colony-forming units (CFU) of $P$. aeruginosa (ATCC 19660). The procedure of corneal infection was performed according to the protocol reported previously (Li et al ., 2020). Sterile saline was used to dissolve the six AR blockers: the $\alpha 1 / 2$ AR blocker Phentolamine $(50 \mathrm{mg} / \mathrm{ml}), \beta 1$-AR blocker Atenolol $(8 \mathrm{mg} / \mathrm{ml}), \beta 2$-AR blocker ICI118551 (5 $\mathrm{mg} / \mathrm{ml}$ ), $\beta 2$-AR blocker Butoxamine (40 mg/ml), $\beta 1 / 2$-AR blocker Timolol $(4.4 \mathrm{mg} / \mathrm{ml})$, or $\beta 3$-AR blocker SR58894A $(5 \mathrm{mg} / \mathrm{ml})$, and $5 \mu \mathrm{L}$ was instilled $30 \mathrm{~min}$ before $P$. aeruginosa inoculation, restarted from $6 \mathrm{~h}$ after infection, and continued for 3 days (six times a day). Control mice were treated with sterile saline. To determine whether ICI118551 prevents ulceration in infected corneas, tobramycin ophthalmic solution (Tobrex) was used to dissolve ICI118551 $(5 \mathrm{mg} / \mathrm{mL})$, and $5 \mu \mathrm{L}$ was instilled into mouse corneas after $P$. aeruginosa inoculation, starting $12 \mathrm{~h}$ after infection and continuing 3 time per day thereafter until $72 \mathrm{~h}$ after infection. The corneal disease was graded using a well-established scale ${ }^{11}$ and scored at 24,48 , and $72 \mathrm{~h}$ post infection.

Determinations of proinflammatory factors and bacterial virulence expression, and Polymorphonuclear Leukocyte (PMN) Infiltration 
As previously described (Gao et al ., 2015), the corneas were excised, minced, and homogenized in $100 \mu \mathrm{L}$ of PBS with a TissueLyser (TissueLyser II, Hilden, Germany). The homogenates were divided into 2 parts. The first part was used for quantitative reverse-transcription polymerase chain reaction (qRT-PCR). Total RNA was extracted using MiniBEST Universal RNA Extraction Kit (Takara, Beijing, China). The cDNA was generated using a PrimeScript RT Reagent Kit, followed by analysis using a real-time PCR kit (Takara) and Rotor-Gene Q systems (Qiagen, Hilden, Germany), based on $\beta$-actin as an internal control for the mice and 16s rRNA for P. aeruginosa. The primers are presented in Supplementary Table 2. The second part was used for the measurements of myeloperoxidase (MPO), tumor necrosis factor- $\alpha$ (TNF- $\alpha$ ), interleukin- $1 \beta$ (IL-1 $\beta$ ), CXCL2, Exotoxin A, lipopolysaccharide (LPS) expression and elastase activity with corresponding enzyme-linked immunosorbent assay (ELISA) kits.

\section{Immunofluorescence and histochemical staining}

As described previously, the corneas were incubated with Ly6G, followed by fluorescein conjugated secondary antibodies, and observed with an Eclipse TE2000-U microscope (Nikon, Tokyo, Japan) (Yoon et al ., 2013). For histological staining, the mouse eyes were fixed in $4 \%$ formalin, embedded in paraffin, and stained with hematoxylin/eosin.

\section{NET Staining}

Sytox green was diluted 1:500 and gently dripped on the surface of the eye, left for 5 minutes, then gently washed off with PBS for 3 times. After that, the cornea was excised, fixed with $4 \%$ paraformaldehyde for 15 minutes, and then washed off, as previously described, with a slight modification (Thanabalasuriar et al ., 2019). The samples were observed using a confocal laser scanning microscope (Carl Zeiss Meditec, Oberkochen, Germany).

\section{Evaluation ofBiofilm Formation}

The corneas were collected, washed, and fixed with $4 \%$ paraformaldehyde for 15 minutes. After that samples were washed and stained with $50 \mu \mathrm{g} / \mathrm{mL}$ fluorescein isothiocyanate-conjugated concanavalin A (FITC-ConA) for 20 minutes, as previously described, with a slight modification (Liet al ., 2020). The biofilm formation was observed using a confocal laser scanning microscope (Carl Zeiss Meditec, Oberkochen, Germany). FITCConA bound to the polysaccharide of the biofilm to emit green fluorescence.

\section{Determination of bacterial load}

Each individual cornea was excised and homogenized in sterile saline. Aliquots $(100 \mathrm{ml})$ of serial dilutions were plated onto Luria-Bertani (LB) agar plates in triplicate and cultured at 37 for $24 \mathrm{~h}$. The bacterial colonies were counted and reported as CFU per cornea.

\section{Growth curve measurement}

P. aeruginosa isolates were grown on LB agar medium at 37, while planktonic cultures were grown with an initial turbidity of $\sim 0.02$ at $600 \mathrm{~nm}$ and shaking at $160 \mathrm{rpm}$. The turbidity of cultures was monitored at 1,2 , $4,6,8,24 \mathrm{~h}$ after bacterial incubation.

\section{Viability staining}

This experiment was performed using the live/dead baclight bacterial viability kit (Thermo Fisher) (Mah et al ., 2003). In brief, concentrate $1 \mathrm{ml}$ of the bacterial culture by centrifugation at 5,000 rpm for $5 \mathrm{~min}$. Remove the supernatant and resuspend the pellet in $1 \mathrm{ml}$ of $0.9 \% \mathrm{NaCl}$ for three times. Mix equal volumes of SYTO 9 and propidium iodide (PI) thoroughly and add $3 \mathrm{ul}$ of the mixture for each $\mathrm{ml}$ of bacterial suspension. After incubate at room temperature in the dark for 15 minutes, the dye mixture was prepared as microscope slides and observed by confocal fluorescent microscopy (Carl Zeiss Meditec, Oberkochen, Germany).

\section{Negative staining}


In brief, concentrate $1 \mathrm{ml}$ of $P$. aeruginosa $24 \mathrm{~h}$ culture and fix by $3 \%$ glutaraldehyde (Tianjin Bodi Chemical Co., Ltd) for $10 \mathrm{~min}$. Then the culture was adsorbed to carbon mesh grids and air dried for 5 min. Next stained with $1 \%$ phosphotungstic acid solution $3 \mathrm{~min}$ and air dried $15 \mathrm{~min}$, then digital images of bacterial were taken with a JEM-1200EX transmission electron microscope (JEOL in Japan).

\section{Flow cytometry}

Five eyes were harvested and minced into pieces. Eye tissue was digested in 2mL RPMI-1640 medium containing $0.5 \mathrm{mg} / \mathrm{mL}$ collagenase VI (Sigma) for 90 minutes at 37 with shaking $(60 \mathrm{rpm}$ ). The digested corneal tissue was then passed through a $100 \mathrm{~mm}$ filter cell strainer (Corning) to remove the debris. The cells were then pelleted by centrifugation at $400 \mathrm{~g}$ for 5 minutes and washed once with $1 \times$ PBS. The cells were subjected to were incubated with Ly6G (1:200) staining for flow cytometric analysis. Cell numbers were measured using a flow cytometer (CytoFLEX LX; Beckman Coulter, CA, USA) according to the manufacturers' instruction. Flow cytometric data were analyzed using CytExpert 2.0 software.

\section{Statistical analysis}

Data were expressed as mean values \pm SD. A commercial statistical/analytical software program (SPSS 20.0; SPSS, Inc., Chicago, IL) was used for statistical analysis. During statistical analysis of bacterial count, bacterial growth curve, MPO assay, flow cytometric analysis, ELISA, and qRT-PCR results, an unpaired two-tailed Student $t$ test was used for comparisons between groups. A nonparametric Mann-Whitney $U$ test was used for clinical scores and viability staining. Differences were considered statistically significant at $P$ values $<.05$.

\section{Results}

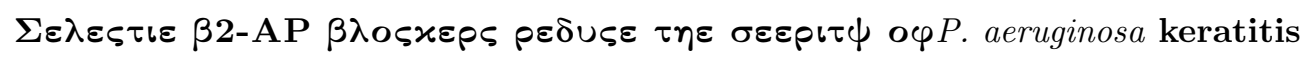

To evaluate the effects of various $\mathrm{AR}$ blockers on the severity of $P$. aeruginosa keratitis, the mice were administrated topically with Phentolamine, Atenolol, ICI118551, Butoxamine, Timolol, and SR58894A $6 \mathrm{~h}$ before bacterial inoculation. As shown in Figure 1A, among the six blockers, only the selective $\beta 2$-AR blockers ICI118551 and Butoxamine exhibited the most apparent reduced severity of $P$. aeruginosa keratitis after both 24 and $72 \mathrm{~h}$. Interestingly, the nonselective $\beta 1 / 2$-AR blocker Timolol caused a mild reduction, while the $\alpha 1 / 2-, \beta 1-$ or $\beta 3$-AR blockers had no significant reduction of disease severity. Moreover, quantitative analysis of clinical scores (Fig. 1B) and corneal bacterial load (Fig. 1C) after $24 \mathrm{~h}$ of infection further confirmed the superior inhibition of $\beta 2$-AR blockers than other blockers on the severity of $P$. aeruginosa keratitis. In addition, the infiltration of inflammatory cells was also reduced apparently through the administration of $\beta 2$ AR blockers than other blockers (Fig. 1D), accompanied with the reduced corneal thickness (Supplementary Fig. 1).

\section{ICI118551 attenuates corneal inflammatory response}

To evaluate the effect of ICI118551 on inflammatory response, neutrophil infiltration and proinflammatory factor expression were investigated at $48 \mathrm{~h}$ after $P$. aeruginosa keratitis infection. Immunofluorescence staining with anti-Ly6G antibody disclosed that neutrophils infiltrated into the anterior stroma in the ICI118551treatment group, compared with full thickness of the cornea infiltration in the vehicle control corneas (Fig. $2 \mathrm{~A})$. The amount and activity of polymorphonuclear neutrophils in the cornea treated with ICI118551 were significantly reduced (Fig. 2B and $\mathrm{C}, P<0.001$ ). The control group showed a typical NETs structure, while the ICI118551 treatment group only showed a small number of independent neutrophils without obvious network structure (Fig. 2D). Furthermore, the ELISA assay (Figure 2E) and RT-PCR (Supplementary Figure 2) revealed that ICI118551 inhibited the expression levels of TNF- $\alpha$, IL-1 $\beta$, and MIP-2 $(P<0.05 ; P<$ $0.01 ; P<0.01)$. These results suggested ICI118551 suppressed the inflammatory response to $P$. aeruginosa keratitis by reducing infiltration of neutrophils and release of inflammatory cytokines.

ICI118551 prophylactic therapy reduced the levels of virulence factors and biofilm formation of $P$. aeruginosa in cornea 
We further explored the level of bacterial virulence in the cornea infected by P. aeruginosa. Compared with the control group, the expression of virulence factor genes was significantly downregulated in the ICI118551-treated corneas (Figure 3A). Besides, the expression levels of P. aeruginosa lipopolysaccharide (LPS), exotoxin A, and elastase were also decreased obviously which were measured by ELISA (Figure 3B; $P<0.05)$. After FITC-ConA staining, the formation of biofilm was observed by confocal microscope. The photographs showed that ICI118551 significantly reduced the density and structure of biofilm (Figure 3C). Above all, it was indicated that the levels of bacterial virulence factors and biofilm formation in the corneas were decreased significantly under the effect of ICI118551.

\section{ICI118551 alleviated damage of the corneal epithelial basement membrane}

As the epithelial basement membrane is considered a biological barrier that bacteria must penetrate (Alarcon et al ., 2009), changes in the infected corneal basement membrane were tested through collagen type IV staining (Figure 4). In the control group, the cornea was severely edematous at $24 \mathrm{~h}$ after infection, and collagen IV staining partially disappeared. Damage to the corneal epithelial basement membrane progressed rapidly, reaching the epithelium basement membrane and deep stroma at $72 \mathrm{~h}$ post infection. The epithelial sheet and part of the stroma were lost, and the corresponding collagen IV staining disappeared. In the ICI118551-treated group, the corneal epithelium was slightly edematous, and staining of the epithelial basement membrane showed mild disorder at $24 \mathrm{~h}$ post infection, followed by rapid recovery. ICI118551 effectively reduced corneal epithelial basement membrane damage.

\section{Bactericidal activity of ICI118551 against $P$. aeruginosain vitro}

To verify the effect of ICI118551 on the growth of $P$. aeruginosa, bacterial growth curve was detected. As shown in Figure 5A, when the bacteria grew up to stable stage, the OD 600 value of the bacterial solution under the influence of ICI118551 decreased significantly compared with that of control. The ICI118551 treatment group showed clearer bacterial liquid at 24 hours post inocubation (Figure 5B). Bacterial survival status of $24 \mathrm{~h}$ culture was detected by viability staining, which shown that there were more dead (red, stained with propidium iodide) than live (green, SYTO9-stained) cells in the group treated with ICI118551(Figure $5 \mathrm{C}, P<0.05)$. Further negative staining electron microscopy verified that bacterial cells under the influence of ICI118551 were dying and on the verge of rupture (Figure 5D). The data above indicated that ICI118551 has significant bactericidal activity against $P$. aeruginosa .

\section{Optimal drug concentration and initial intervention time of ICI118551}

Considering the significant effect of ICI118551 on P. aeruginosa keratitis, we further explored the optimal drug concentration and initial intervention time. The effects of three different concentrations of ICI118551 were evaluated on infected corneas, including $1 \mathrm{mg} / \mathrm{mL}, 2 \mathrm{mg} / \mathrm{mL}$ and $5 \mathrm{mg} / \mathrm{mL}$ (maximum concentration of solution in sterile saline). Representative photographs and clinical scores quantified 72 hours after infection showed that cornea treated with $5 \mathrm{mg} / \mathrm{mL}$ ICI118551 showed higher transparency and lower clinical scores (Figure 6A and B). Moreover, three different administration time of ICI118551 $(5 \mathrm{mg} / \mathrm{mL})$ were also optimized. Based on the data of three different administration time $(0.5 \mathrm{~h}, 6 \mathrm{~h}$, and $12 \mathrm{~h}$ after bacterial incubation), it was indicated that the earlier the intervention time, the better the efficacy of ICI118551 against $P$. aeruginosa keratitis of mice with no significant effect on intraocular pressure (Figure $6 \mathrm{C}$ and Supplementary Figure 3).

\section{ICI118551 adjunct therapy with Tobrex reduced the severity of $P$. aeruginosakeratitis}

The aforementioned results suggested that ICI118551 is effective in reducing P. aeruginosa keratitis. Up to date the best treatment for bacterial keratitis remains topical antibiotic (Austin et al ., 2017). We then investigated the effect of topically applied ICI118551 combined with antibiotics on the P. aeruginosakeratitis. We chose 12 hours after infection as a starting point to treat $P$. aeruginosa keratitis with topical Tobrex in the presence or absence of ICI118551 (5 mg/mL). Figure 7 showed the representative micrographs of infected corneas 12, 24, 48 and $72 \mathrm{~h}$ after infection. Before the intervention, the infected eyes in the two groups exhibited some slight opacification and subepithelial haze. While Tobrex-treated eyes exhibited topical dense 
opacification and subepithelial haze, the eyes treated with ICI118551 as adjunctive therapy were mostly clear with some areas of slight opacity (Figure 7A). A significant difference was observed $72 \mathrm{~h}$ after infection between the clinical scores of corneas cotreated with ICI118551 and Tobrex and those of corneas treated with Tobrex (Figure 7B). Consistent with reduced corneal infection, topical ICI118551 treatment significantly reduced bacterial load, compared with Tobrex alone (Figure 7C; $P<0.001$ ).

\section{Discussion}

Previous studies reported the use of $\beta 2$-AR blockers as therapy for pulmonary, cardiovascular, and other diseases (Brodde, 2007; Leineweber \& Heusch, 2009; O'Neill et al ., 2020; Wenzel et al ., 2009). However, the effect of $\beta 2$-AR blockers on cornea was unclear (Michel, Bond \& Summers, 2019). Our study showed that the prophylactic use of the highly selective $\beta 2$-AR blocker ICI118551 suppressed the severity of $P$. aeruginosa keratitis by reducing neutrophil recruitment, NETs formation, and cytokines release. It also inhibited the formation of biofilm and the production of bacterial virulence factors in cornea. Further bacterial culture in vitro confirmed ICI118551 has significantly antibacterial activity against $P$. aeruginosa . Subsequently, the topical ICI118551 treatment combined with Tobrex 12h after infection greatly reduced the severity of $P$. aeruginosa keratitis compared with Tobrex alone, suggesting an adjunct therapy role of ICI118551. To our knowledge, this is the first study to explore the role of $\beta$-AR blockers on bacterial keratitis.

Our previous studies have confirmed that extended CL wear could elevate corneal NE content, which promotes the pathogenesis of CL-induced $P$. aeruginosa keratitis in mice. Here in this study, we explored the effects of different AR blockers on the severity of $P$. aeruginosa keratitis in mice. NE and AR blockers have been shown to modulate bacterial infection by interaction with bacteria and immune response (Chavan et al ., 2017; Padro \& Sanders, 2014; Vaziraniet al ., 2015; Xue et al ., 2018). Stimulation of the $\beta 2-A R$ pathway was found to be detrimental for host survival to cytomegalovirus infection, while $\beta 2$-AR deficient mice exhibited a higher resistance to cytomegalovirus infection (Wieduwild et al ., 2020). Accordingly, we found that $\beta 2$-AR blockers have a more obvious effect on reducing the severity of keratitis among six blockers, and ICI118551 has the most significant effect.

Recent evidence suggested ICI118551 significantly inhibited the mobility by binding to $\beta 2$-AR of neutrophil cell membrane (Reinartz et al ., 2016). Moreover, in cornea, $\beta 2$-AR blockers could inhibit the rate and number of neutrophils trafficking to the injured cornea following corneal abrasion by binding to the $\beta 2$-AR (Diaz-Salazar et al ., 2020; Wieduwild et al ., 2020; Xue et al ., 2018), thus regulating the release of inflammatory mediators such as IL-6, IL-17A and TNF- $\alpha$, inactivation nuclear factor- $\varkappa \mathrm{B}(\mathrm{NF}-\varkappa \mathrm{B})$ signaling pathway and reducing host inflammation response. The inflammatory mediators mentioned above would induce inflammation via NF- $x$ B signaling pathway (McClellan et al ., 2006) and initiate bacterial eradication during the pathogenic process of $P$. aeruginosa infection. Based on our data, the neutrophil infiltration and NETs formation were inhibited from the effect of ICI118551. Indeed, the expression of inflammatory cytokines such as MIP-2, TNF- $\alpha$ and IL-1 $\beta$ were also downregulated along with the suppression of $P$. aeruginosa infection. This constitutes an important indication that ICI118551 reduced the severity of $P$. aeruginosa keratitis via $\beta 2$-AR pathway and regulation of infiltration of neutrophils.

As reported previously, NE could promote the adhesion and biofilm formation of P. aeruginosa. The formation of biofilms by $P$. aeruginosa is thought to increase persistence and antibiotic resistance during infection and like to the release of virulence factors, regulated by complicated signaling systems such as las and rhlquorum sensing systems (Flemming \& Wingender, 2010; Lee \& Zhang, 2015; Riquelme et al ., 2020). Herein, our data show that the $\beta 2$-AR blocker ICI118551 reduced the formation of biofilm in the corneas, which is beneficial to the phagocytosis of bacteria by neutrophils. Additionally, recent study reported the non-selective $\beta$-blocker, carvedilol, exhibited obvious antibacterial activity by changing the percentage of membrane phospholipids and fatty acids, and influencing the permeability of bacterial cell membranes (Zawadzka et al ., 2018). While in this study, we confirmed that $\beta 2$-AR blocker ICI118551, also has a significant bactericidal effect on $P$. aeruginosa, which can obviously inhibit bacterial growth and the expression levels of virulence factors in cornea. The antibacterial activity of ICI118551 was verified by the staining tests of cell membranes permeability of $P$. aeruginosa incubated with ICI118551 in vitro. This is 
further indication that ICI118551 not only participated in the regulation of host neutrophil mobility, but also has a non-negligible inhibitory effect on $P$. aeruginosa signal pathways.

In view of the significant inhibition of ICI118551 on $P$. aeruginosa keratitis, we further explored the optimal drug concentration, initial intervention time, and its potential influence on intraocular pressure. From the quantification of the clinical scores, the optimal concentration was $5 \mathrm{mg} / \mathrm{ml}$, the initial intervention time was as earlier as the bacterial inoculation in the cornea. From the dynamic evaluation of intraocular pressure, ICI118551 had no significant effect on this parameter during or after topical administration.

Although invasive $P$. aeruginosa can be readily eliminated by topical antibiotics, persistent inflammation may lead to the progression of corneal ulcer (Gao et al ., 2015). Herein, using the established murine model of bacterial keratitis, we examined the therapeutic potential for ICI118551 as an adjunct therapy to Tobrex. We noted that the infected cornea treated with ICI118551+Tobrex exhibited the better improvement in disease severity compared with Tobrex alone. Combined with minimal side effects of topical administration of ICI118551, it merits further development of ICI118551 in the treatment of $P$. aeruginosa keratitis.

Collectively, this study demonstrated that the highly selective $\beta 2$-AR blocker ICI118551 suppressed P. aeruginosa keratitis and alleviate corneal damage of mice. It is suggested that ICI118551 may represent a kind of potential prophylactic and therapeutic approach to control the corneal and other mucosalP. aeruginosa infection in the future.

\section{Acknowledgements}

We thank Ting Liu, Xia Qi, and Li Ma for their excellent technical assistance.

\section{References}

Alarcon I, Kwan L, Yu C, Evans DJ, \& Fleiszig SM (2009). Role of the corneal epithelial basement membrane in ocular defense against Pseudomonas aeruginosa. Infection and immunity 77:3264-3271.

Austin A, Lietman T, \& Rose-Nussbaumer J (2017). Update on the management of infectious keratitis. Ophthalmology 124:1678-1689.

Brodde OE (2007). Beta-adrenoceptor blocker treatment and the cardiac beta-adrenoceptor-G-protein(s)adenylyl cyclase system in chronic heart failure. Naunyn-Schmiedeberg's archives of pharmacology 374:361372.

Carnt N, Samarawickrama C, White A, \& Stapleton F (2017). The diagnosis and management of contact lens-related microbial keratitis. Clinical \& experimental optometry 100: 482-493.

Chavan SS, Pavlov VA, \& Tracey KJ (2017). Mechanisms and therapeutic relevance of Neuro-immune communication. Immunity 46: 927-942.

Cunrath O, Meinel DM, Maturana P, Fanous J, Buyck JM, Saint Auguste P, et al. (2019). Quantitative contribution of efflux to multi-drug resistance of clinical Escherichia coli and Pseudomonas aeruginosa strains. EBioMedicine 41: 479-487.

Diaz-Salazar C, Bou-Puerto R, Mujal AM, Lau CM, von Hoesslin M, Zehn D, et al. (2020). Cell-intrinsic adrenergic signaling controls the adaptive NK cell response to viral infection. The Journal of experimental medicine 217.

Fleiszig SMJ, Kroken AR, Nieto V, Grosser MR, Wan SJ, Metruccio MME, et al. (2020). Contact lensrelated corneal infection: Intrinsic resistance and its compromise. Prog Retin Eye Res 76:100804.

Flemming HC, \& Wingender J (2010). The biofilm matrix. Nature reviews Microbiology 8: 623-633.

Gao N, Kumar A, \& Yu FS (2015). Matrix metalloproteinase-13 as a target for suppressing corneal ulceration caused by Pseudomonas aeruginosa infection. The Journal of infectious diseases 212:116-127.

Hazlett LD (2004). Corneal response to Pseudomonas aeruginosainfection. Prog Retin Eye Res 23: 1-30. 
Lakhundi S, Siddiqui R, \& Khan NA (2017). Pathogenesis of microbial keratitis. Microbial Pathogenesis 104: $97-109$.

Lee J, \& Zhang L (2015). The hierarchy quorum sensing network in Pseudomonas aeruginosa. Protein \& cell 6: 26-41.

Lee JE, Sun Y, Gjorstrup P, \& Pearlman E (2015). Inhibition of corneal inflammation by the resolvin E1. Investigative ophthalmology \& visual science 56: 2728-2736.

Leineweber K, \& Heusch G (2009). $\beta 1$ - and $\beta 2$-Adrenoceptor polymorphisms and cardiovascular diseases. British journal of pharmacology 158: 61-69.

Li J, Ma X, Zhao L, Li Y, Zhou Q, \& Du X (2020). Extended contact lens wear promotes corneal norepinephrine secretion and Pseudomonas aeruginosa infection in mice. Investigative ophthalmology \& visual science 61: 17 .

Ma L, Wang S, Wang D, Parsek MR, \& Wozniak DJ (2012). The roles of biofilm matrix polysaccharide Psl in mucoid Pseudomonas aeruginosa biofilms. FEMS immunology and medical microbiology 65: 377-380.

Ma X, Wang Q, Song F, Li Y, Li J, Dou S, et al. (2020). Corneal epithelial injury-induced norepinephrine promotes Pseudomonas aeruginosa keratitis. Experimental eye research 195: 108048.

Mah TF, Pitts B, Pellock B, Walker GC, Stewart PS, \& O'Toole GA (2003). A genetic basis for Pseudomonas aeruginosa biofilm antibiotic resistance. Nature 426: 306-310.

McClellan SA, Huang X, Barrett RP, Lighvani S, Zhang Y, Richiert D, et al. (2006). Matrix metalloproteinase-9 amplifies the immune response to Pseudomonas aeruginosa corneal infection. Investigative ophthalmology \& visual science 47: 256-264.

Michel MC, Bond RA, \& Summers RJ (2019). Adrenoceptors-New roles for old players. British journal of pharmacology 176: 2339-2342.

O'Neill E, Yssel JD, McNamara C, \& Harkin A (2020). Pharmacological targeting of $\beta(2)$-adrenoceptors is neuroprotective in the LPS inflammatory rat model of Parkinson's disease. British journal of pharmacology 177: 282-297.

Oka N, Suzuki T, Ishikawa E, Yamaguchi S, Hayashi N, Gotoh N, et al. (2015). Relationship of virulence factors and clinical features in keratitis caused by Pseudomonas aeruginosa. Investigative ophthalmology \& visual science 56: 6892-6898.

Okada F, Kobayashi M, Tanaka H, Kobayashi T, Tazawa H, Iuchi Y, et al. (2006). The role of nicotinamide adenine dinucleotide phosphate oxidase-derived reactive oxygen species in the acquisition of metastatic ability of tumor cells. The American journal of pathology 169:294-302.

Padro CJ, \& Sanders VM (2014). Neuroendocrine regulation of inflammation. Semin Immunol 26: 357-368.

Papayannopoulos V (2018). Neutrophil extracellular traps in immunity and disease. Nat Rev Immunol 18: 134-147.

Pearlman E, Johnson A, Adhikary G, Sun Y, Chinnery HR, Fox T, et al. (2008). Toll-like receptors at the ocular surface. Ocul Surf 6: 108-116.

Reinartz MT, Wetzke M, Happle C, Kälble S, Scherer R, Kabesch M, et al. (2016). Neutrophilic superoxide production can assess pharmacological and pharmacogenetic $\beta$-adrenoreceptor effects. Allergy 71: 1223-1227.

Riquelme SA, Liimatta K, Wong Fok Lung T, Fields B, Ahn D, Chen D, et al. (2020). Pseudomonas aeruginosa utilizes host-derived itaconate to redirect its metabolism to promote biofilm formation. Cell Metab. 
Subedi D, Vijay AK, \& Willcox M (2018). Overview of mechanisms of antibiotic resistance in Pseudomonas aeruginosa : an ocular perspective. Clinical \& experimental optometry 101: 162-171.

Suzuki T, Okamoto S, Oka N, Hayashi N, Gotoh N, \& Shiraishi A (2018). Role of pvdE pyoverdine synthesis in Pseudomonas aeruginosakeratitis. Cornea 37 Suppl 1: S99-s105.

Thanabalasuriar A, Scott BNV, Peiseler M, Willson ME, Zeng Z, Warrener P, et al. (2019). Neutrophil extracellular traps confinePseudomonas aeruginosa ocular biofilms and restrict brain invasion. Cell host \& microbe.

Vazirani J, Wurity S, \& Ali MH (2015). Multidrug-resistantPseudomonas aeruginosa keratitis: risk factors, clinical characteristics, and outcomes. Ophthalmology 122: 2110-2114.

Wenzel D, Knies R, Matthey M, Klein AM, Welschoff J, Stolle V, et al. (2009). beta(2)-adrenoceptor antagonist ICI 118,551 decreases pulmonary vascular tone in mice via a G(i/o) protein/nitric oxide-coupled pathway. Hypertension (Dallas, Tex : 1979) 54:157-163.

Wieduwild E, Girard-Madoux MJ, Quatrini L, Laprie C, Chasson L, Rossignol R, et al. (2020). beta2adrenergic signals downregulate the innate immune response and reduce host resistance to viral infection. The Journal of experimental medicine 217.

Willcox MD (2007). Pseudomonas aeruginosa infection and inflammation during contact lens wear: a review. Optometry and vision science : official publication of the American Academy of Optometry 84:273-278.

Xue Y, He J, Xiao C, Guo Y, Fu T, Liu J, et al. (2018). The mouse autonomic nervous system modulates inflammation and epithelial renewal after corneal abrasion through the activation of distinct local macrophages. Mucosal immunology 11: 1496-1511.

Yang H, Biermann MH, Brauner JM, Liu Y, Zhao Y, \& Herrmann M (2016). New insights into neutrophil extracellular traps: mechanisms of formation and role in inflammation. Frontiers in immunology 7:302.

Yoon GS, Dong C, Gao N, Kumar A, Standiford TJ, \& Yu FS (2013). Interferon regulatory factor-1 in flagellin-induced reprogramming: potential protective role of CXCL10 in cornea innate defense against Pseudomonas aeruginosa infection. Investigative ophthalmology \& visual science 54: 7510-7521.

Yue L, Xie Z, Li H, Pang Z, Junkins RD, Tremblay ML, et al.(2016). Protein tyrosine phosphatase-1B negatively impacts host defense against Pseudomonas aeruginosa infection. The American journal of pathology 186: $1234-1244$.

Zawadzka K, Bernat P, Felczak A, Rozalska S, \& Lisowska K (2018). Antibacterial activity of high concentrations of carvedilol against Gram-positive and Gram-negative bacteria. International journal of antimicrobial agents 51: 458-467.

Zhao Y, Guo Q, Dai X, Wei X, Yu Y, Chen X, et al. (2019). A biomimetic non-antibiotic approach to eradicate drug-resistant infections. Advanced materials (Deerfield Beach, Fla) 31:e1806024.

Figure legends

Figure 1. Effects of different AR blockers on $\boldsymbol{P}$. aeruginosa keratitis. AR blockers or sterile saline (control) were topically applied respectively to the mice corneas $0.5 \mathrm{~h}$ before corneal infection. (A) Representative photographs of corneas at $24 \mathrm{~h}$ and $72 \mathrm{~h}$ after bacterial inoculation. (B) The corneal clinical scores at $72 \mathrm{~h}$ after bacterial inoculation. (C) The bacterial load in each individual cornea $24 \mathrm{~h}$ after bacterial inoculation was counted $\left(*, P<.05,{ }^{* *}, P<.01,{ }^{* * *}, P<.001 ; \mathrm{n}=5\right)$. (D) Representative photomicrographs stained with hematoxylin/eosin at $24 \mathrm{~h}$ after bacterial inoculation. Abbreviation: E, epithelium.

Figure 2. ICI118551 attenuated neutrophil infiltration, NETs formation and the proinflammatory cytokine levels. The infected corneas were excised $48 \mathrm{~h}$ after infection and subjected to immunofluorescence staining with the Ly6G antibody (A), flow cytometric quantification of the cumulative 
neutrophil (Ly6G+) percentage among total leukocytes (B), MPO activity assay (C), NETs staining (D), and a proinflammatory cytokine ELISA assay $(\mathrm{E})\left(^{*}, P<.05,{ }^{* *}, P<.01,{ }^{* * *}, P<.001 ; \mathrm{n}=5\right)$.

Figure 3. ICI118551 reduced the levels of virulence factors and biofilm formation of $P$. aeruginosa in corneas. Mice corneas were excised at $24 \mathrm{~h}$ after infection and subjected to qRT-PCR (A), ELISA assay (B) and biofilm staining (C). The expression level of flagellin related genes figD $/ E / L$, elastase related genes $\operatorname{las} A / B$, and biofilm related gene $p s l B$ were detected. The content of LPS, exotoxin A, and elastase activity were measured $\left(*, P<.05,{ }^{* *} P<.01 ; \mathrm{n}=5\right)$.

Figure 4. ICI118551 reduced corneal epithelial basement membrane damage (E). The infected corneas were subjected to immunofluorescence staining analysis using anti-collagen IV to illuminate the basement membrane (arrowheads) at 24, 48, and $72 \mathrm{~h}$ after infection. Abbreviation: E, epithelium.

Figure 5. Bactericidal activity of ICI118551 against $P$. aeruginosa in vitro. The effect of ICI118551 on the growth of $P$. aeruginosa in vitro was detected. (A) Bacterial growth curve. The turbidity of cultures was monitored at $1,2,4,6,8,24 \mathrm{~h}$ after bacterial incubation $\left(*, P<.05,{ }^{* * *}, P<.001\right)$. (B) Comparison of $P$. aeruginosa 24-hour culture. (C) Viablity staining results of $P$. aeruginosa 24-hour culture. Red, stained with propidium iodide; green, stained with SYTO9. (D) Negative staining results of P. aeruginosa 24-hour culture. Black bacterial cells represent a complete cell structure, while gray indicates that cells are dissolving (arrow).

Figure 6. Determination of the optimal drug concentration and optimal initial intervention time. The effect of three different concentration $(1 \mathrm{mg} / \mathrm{ml}, 2 \mathrm{mg} / \mathrm{ml}$, and $5 \mathrm{mg} / \mathrm{ml})$, and three different administration time $(0.5,6$ and $12 \mathrm{~h}$ post infection) of ICI118551 were evalued. Representative images of slit lamp microscopy (A) and clinical scores (B and C) at 24, 48, and $72 \mathrm{~h}$ after bacterial inoculation were shown. The bacterial challenge was followed by ICI118551 treatment to contrast the anti-inflammatory effect at different initial intervention times: $0.5,6$ and $12 \mathrm{~h}$ represent the time intervals between the two events $\left(^{*}\right.$, $\left.P<.05,{ }^{* *}, P<.01 ; \mathrm{n}=5\right)$.

Figure 7. ICI118551 adjunct therapy combined with Tobrex significantly reduced the severity of $\boldsymbol{P}$. aeruginosa keratitis.ICI118551 combined Tobramycin or Tobramycin was applied topically start from $12 \mathrm{~h}$ after infection and 3 times per day. (A) Representative photographs and clinical scores (B) of the tobramycin and ICI118551 combined tobramycin treated corneas at $12 \mathrm{~h}$ and $72 \mathrm{~h}$ after infection. (C) Bacterial counting of corneas $72 \mathrm{~h}$ after infection $\left(*, P<.05,{ }^{* *}, P<.01 ; \mathrm{n}=5\right)$.

Supplementary Figure 1. ICI118551 reduced pathological changes in the infected cornea. The thickness of sections stained with hematoxylin/eosin of the infected cornea at $24 \mathrm{~h}$ after bacterial inoculation were shown $\left(*, P<.05,{ }^{*}, P<.01 ; \mathrm{n}=5\right)$.

Supplementary Figure 2. ICI118551 negatively regulated the expression of proinflammatory cytokine genes. The expression levels of proinflammatory cytokines, including MIP-2, TNF- $\alpha$, and IL-1 $\beta$, were measured by a qRT-PCR $\left(* *, P<.01,{ }^{* * *}, P<.001 ; \mathrm{n}=5\right)$.

Supplementary Figure 3. The effect of ICI118551 on intraocular pressure. ICI118551 at a concentration of $5 \mathrm{mg} / \mathrm{ml}$ or sterile saline (control) was instilled into mice corneas and applied six times/d for $72 \mathrm{~h}$. Intraocular pressure was then detected using a TonoLab tonometer (TioLat, Helsinki, Finland). 

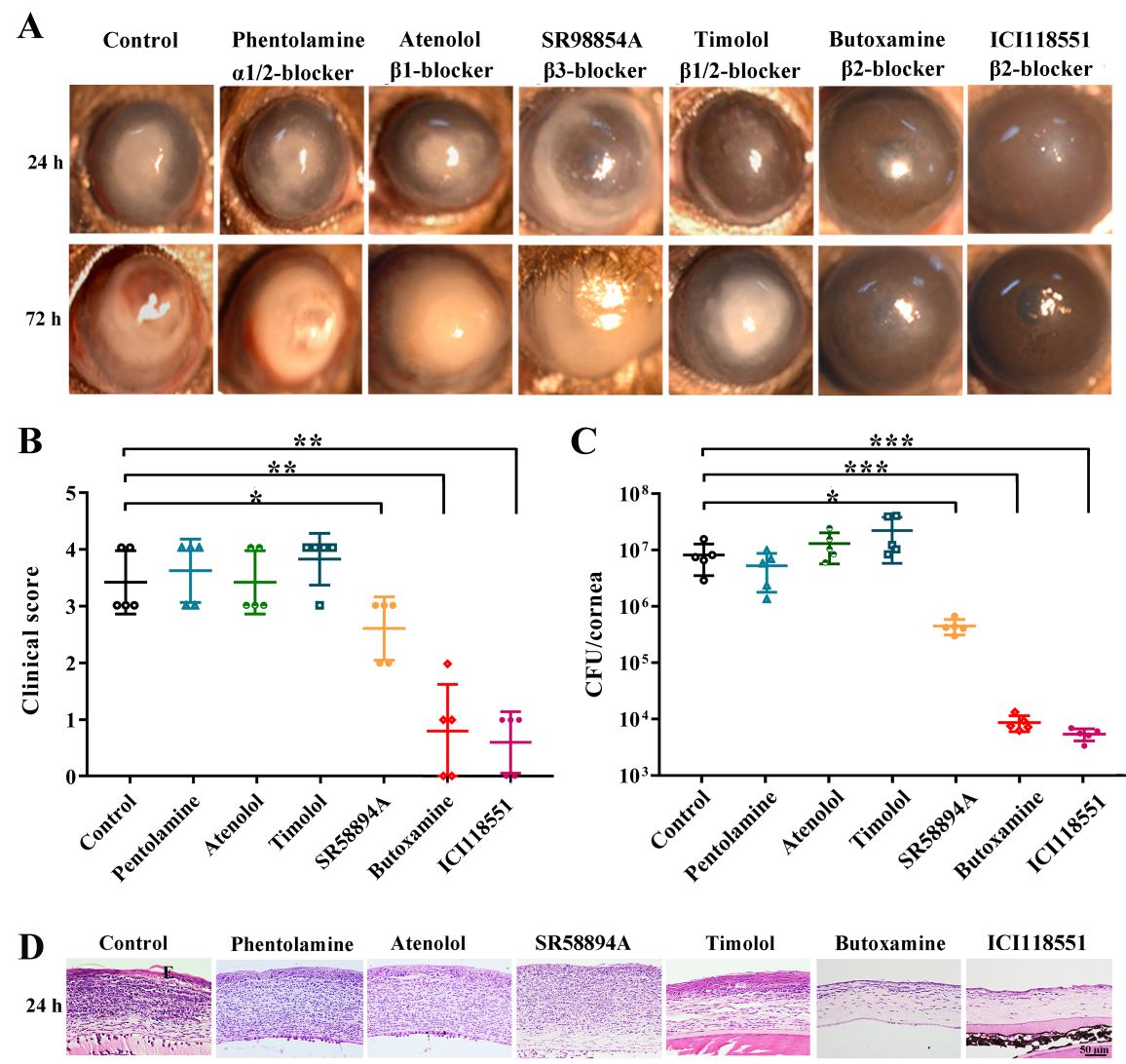

Figure 1
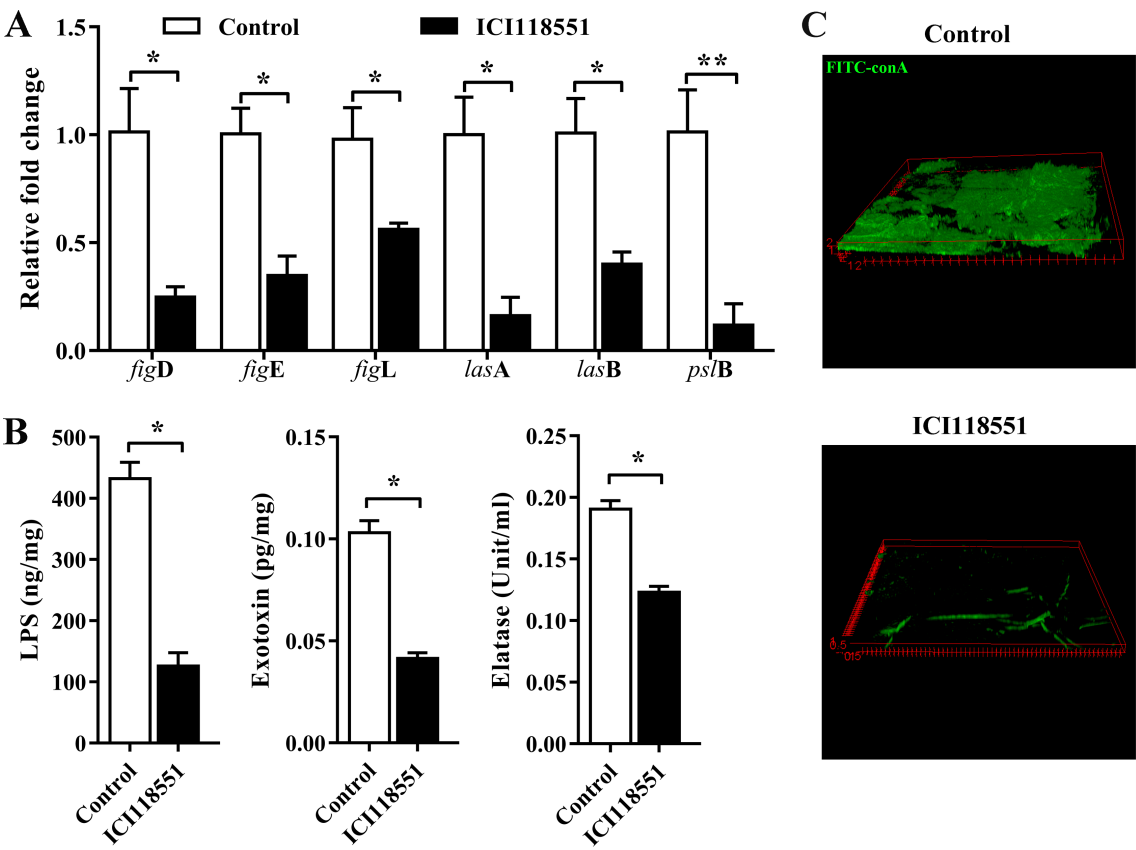

ICI118551

Figure 3 


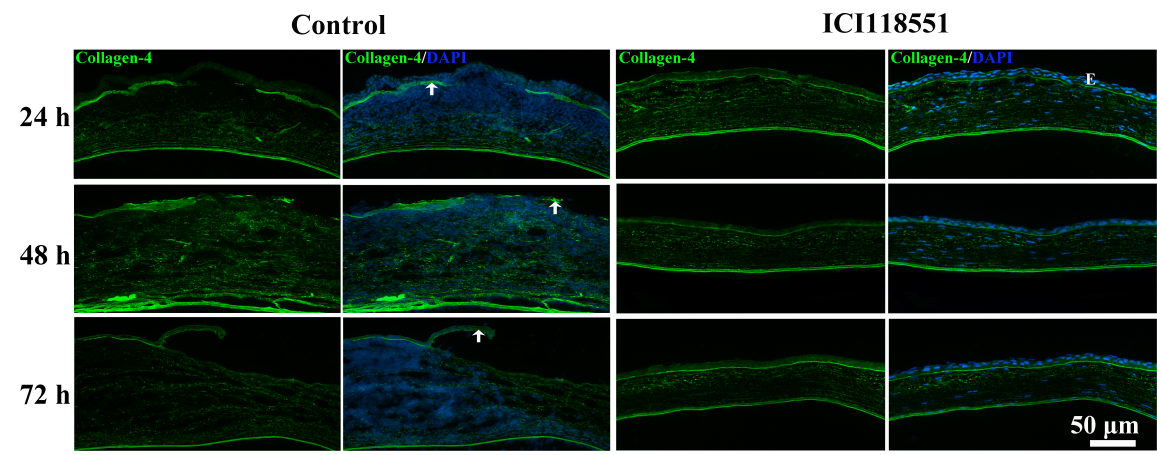

Figure 4

A

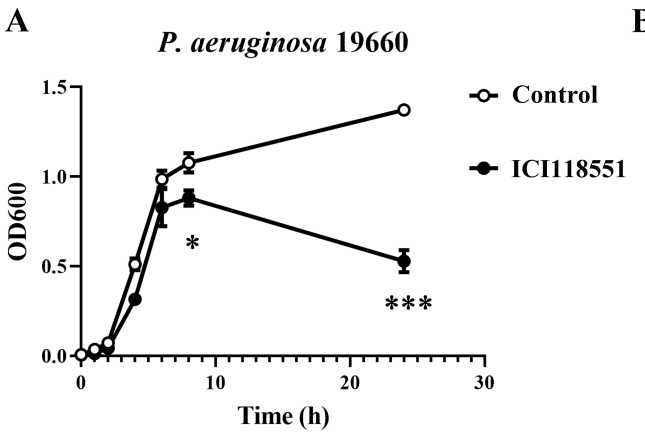

C
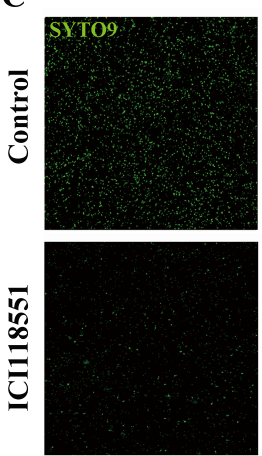

B

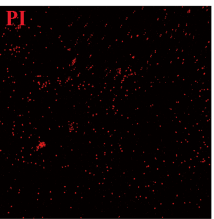

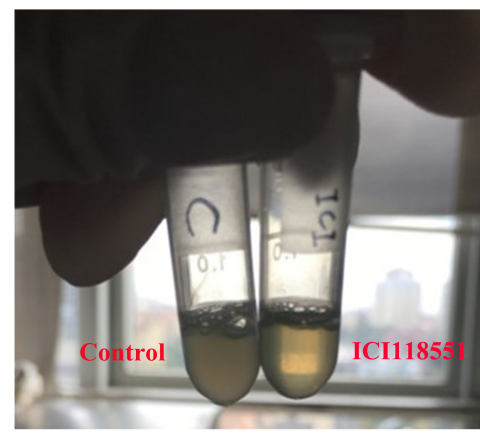

D
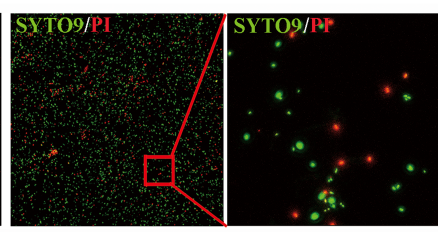

0
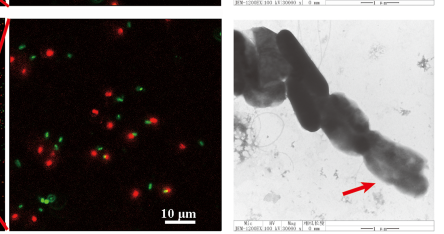

Figure 5 

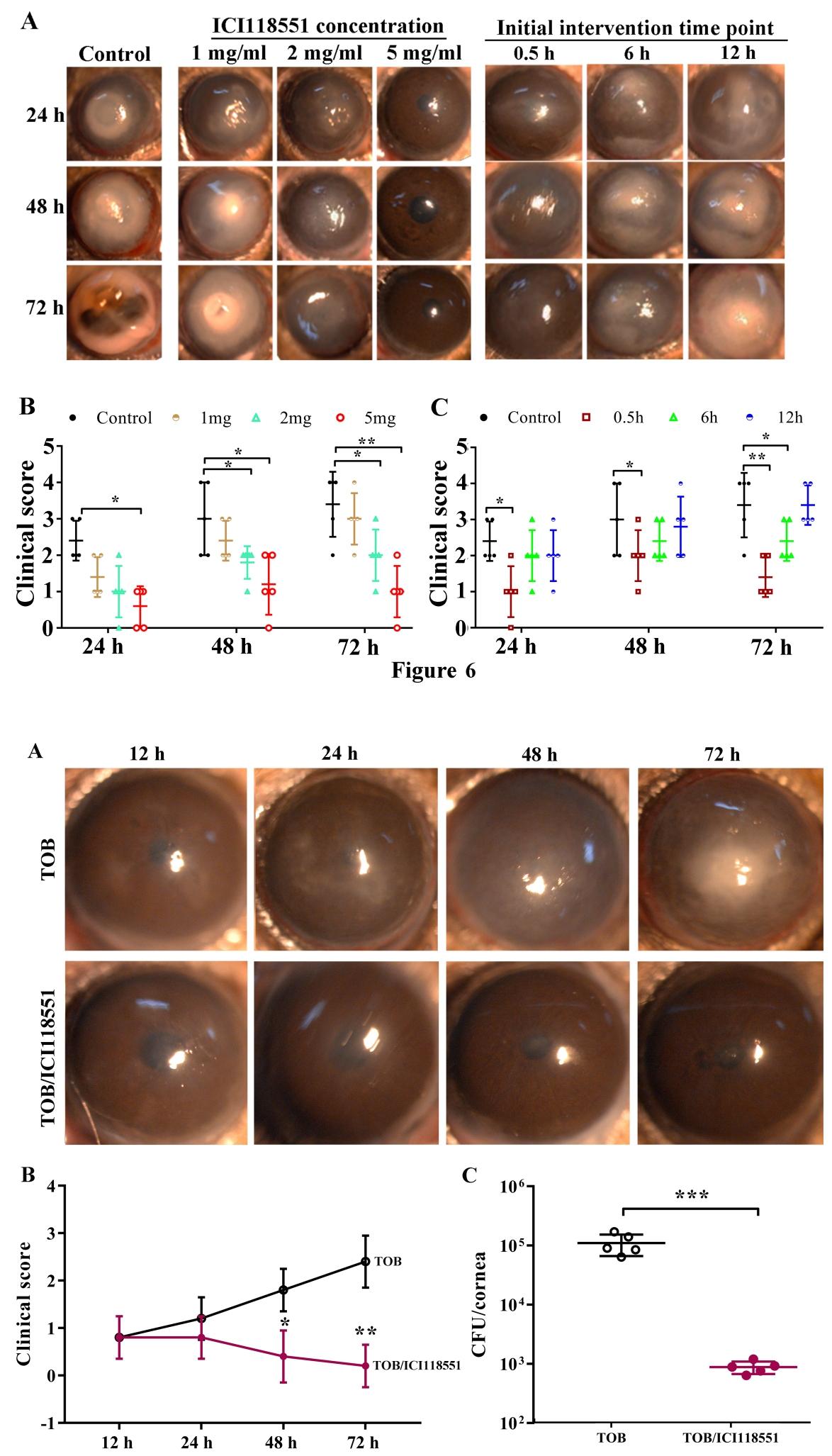

Figure 7 


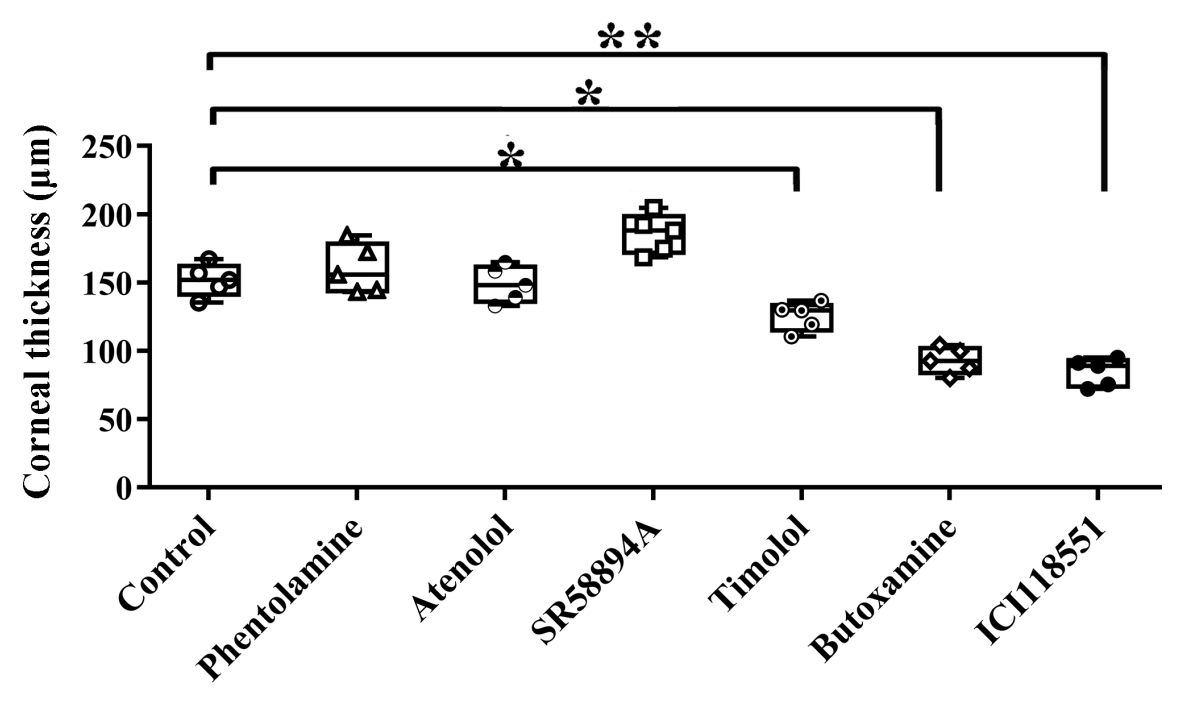

Supplementary Figure 1

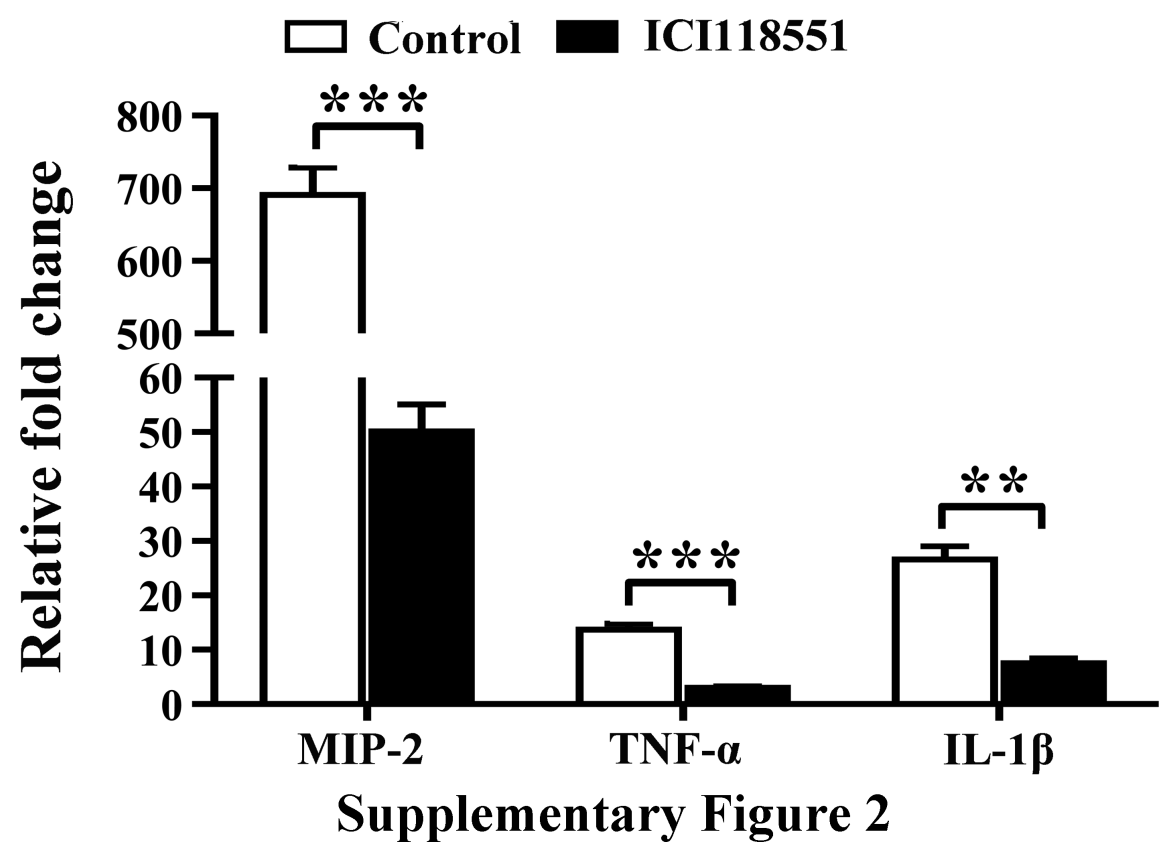




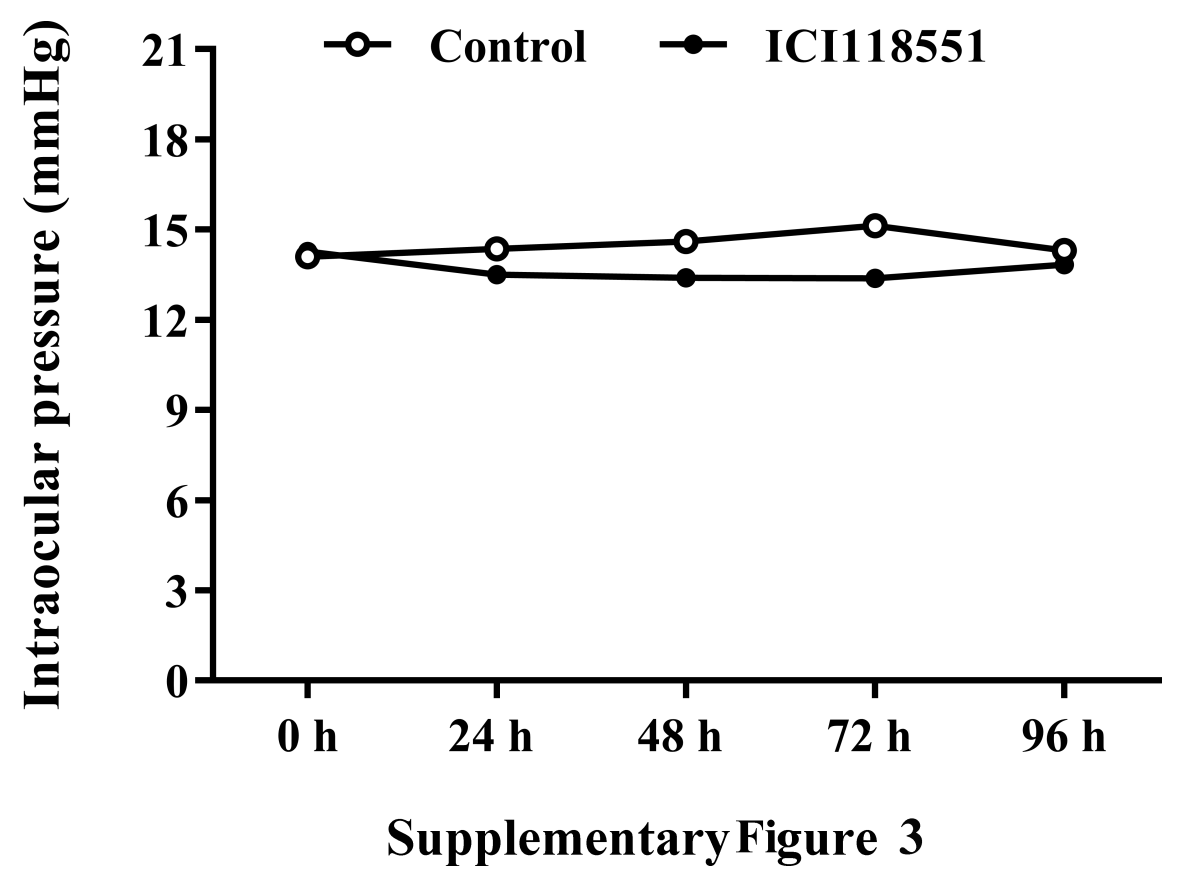

Hosted file

Supplementary Tables-manuscript.pdf available at https://authorea.com/users/364344/articles/ 484818-\%CE\%B22-adrenoceptor-blocker-ici118551-attenuates-pseudomonas-aeruginosa-cornealinfection-in-mice
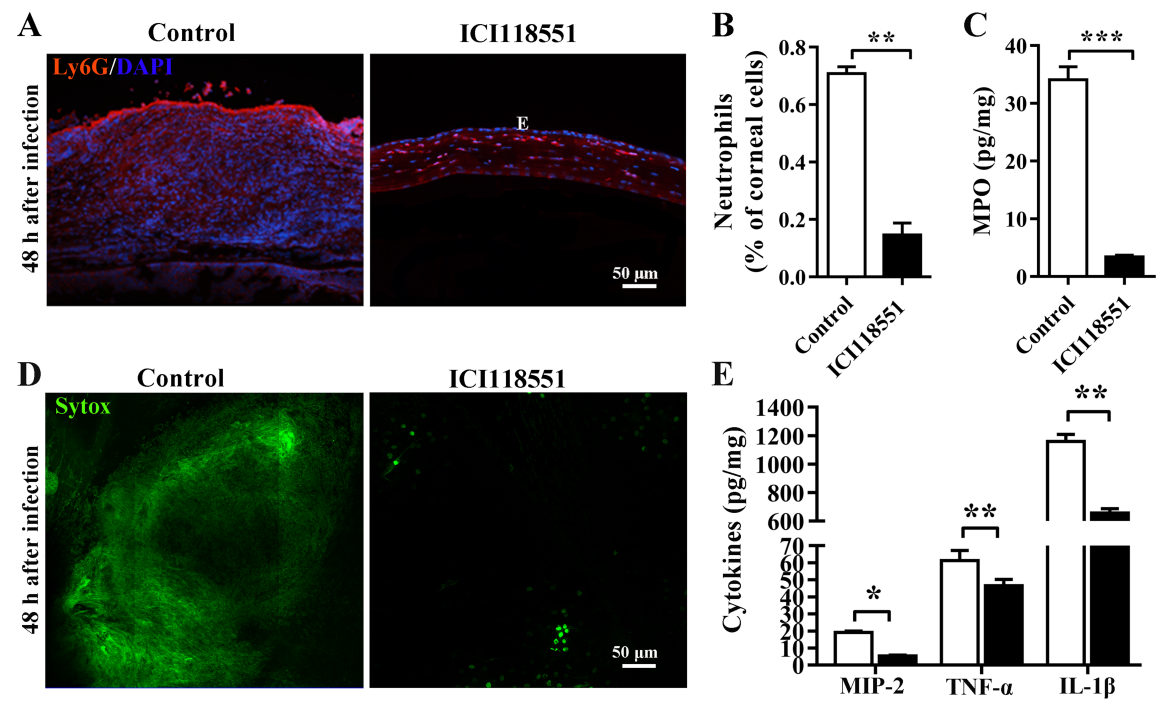

Figure 2 